\title{
Neuromuscular disorder as initial manifestation of secondary hyperparathyroidism - a case report
}

\author{
Karim Hajjar, Tim Hagenacker \\ Department of Neurology, University Hospital Essen, Germany \\ This article is distributed under the terms of the Creative Commons Attribution Noncommercial License (CC BY-NC 4.0) which permits
} any noncommercial use, distribution, and reproduction in any medium, provided the original author(s) and source are credited.

\begin{abstract}
This case report describes a young woman who presented with slowly progressing weakness of the proximal limb muscles and slight decrease of muscle tone but otherwise unremarkable neurological history. Diagnostic workup revealed fatty degeneration of the girdle muscles and an excessive increase of the parathyroid hormone as a result of severe vitamin D deficiency. Secondary hyperparathyroidism was diagnosed, and neurological deficits resolved after treatment of the underlying endocrinopathy.
\end{abstract}

Key Words: neuromuscular disorder, endocrine myopathy, hyperparathyroidism

Eur J Transl Myol 27 (1): 40-42

Hyperparathyroidism (HPT) is defined as oversecretion of parathyroid hormone (PTH) which regulates calcium and phosphate levels. While primary hyperparathyroidism (PHPT) results from inappropriate overactivity of the parathyroid glands, secondary hyperparathyroidism (SHPT) is the result of increased PTH secretion to compensate hypocalcemia (low blood calcium levels). Alkaline phosphatase (ALP) levels are usually elevated in both PHPT and SHPT as a result of increased bone resorption. SHPT often occurs in patients with vitamin D deficiency and chronic kidney failure. Asymptomatic disease is common, and clinical signs are mainly due to bone resorption, predominantly manifesting as bone syndromes, e.g. osteomalacia or osteodystrophy.

\section{Case Report}

We discuss the case of a 25-year old woman suffering from progressive limb weakness and clinical presentation of a proximal tetraparesis with slightly reduced muscular tone and mild Trendelenburg's sign. She has had difficulties in walking longer distances since the age of 14 when she underwent corrective osteotomy for congenital genu valgum. However during the last years she noticed a newly developed decrease in tone and strength of the proximal muscles, predominantly affecting the lower limbs, especially when climbing stairs. Muscular weakness had slowly progressed, being most prominent in the evening hours. For the past 6 months she had been dependent on walking aids (canes or crutches). Moreover she reported of exertion induced myalgia. Family history at this time was unremarkable.
Clinical examination showed a symmetric proximal tetraparesis particular of the limb girdle muscles (MRC grade 4+ / 5 on arm elevation, rotation and abduction and 4 / 5 on hip and knee flexion and extension on both sides). Strength of the distal muscles was unremarkable. Muscle tone was slightly decreased. There were no relevant muscular atrophies. Tendon reflexes were normal. Examination of gait revealed slight bilateral Trendelenburg's sign. History and clinical features were consistent with signs of a neuromuscular disorder.

On diagnostic work-up, both cranial and spinal MRI scans were unremarkable, as well as the lumbar punction. ECG and echocardiogram showed no abnormalities. Electrophysiological examination showed neither signs of peripheral sensory or motoric neuropathy nor of central nervous disorders, however EMG revealed myopathic changes in the proximal muscles of the upper and lower limbs with few myotonic discharges. MRI of the thighs revealed fatty degeneration of the gluteal and quadriceps muscles (Figs. 1 and 2). X-ray of pelvis, hip and knee joints showed no osseous destruction but moderate signs of osteoporosis. Computed tomography of the spine and pelvis measuring bone mineral density showed a $\mathrm{Z}$ score of -4.0 compatible with generalized osteoporosis. Blood tests showed serum creatininkinase and myoglobine within normal range, however Alkaline phosphatase (ALP) was elevated (254 U/l, ref. < 104). Thyroid function tests were unremarkable including thyroid antibodies. Screening of autoantibodies showed elevation of antinuclear antibody (ANA) titer (1:320, ref. $<1: 80)$ and positive antibodies against Sjoegren's syndrom A (SSA-AB), whereas all other antibodies 


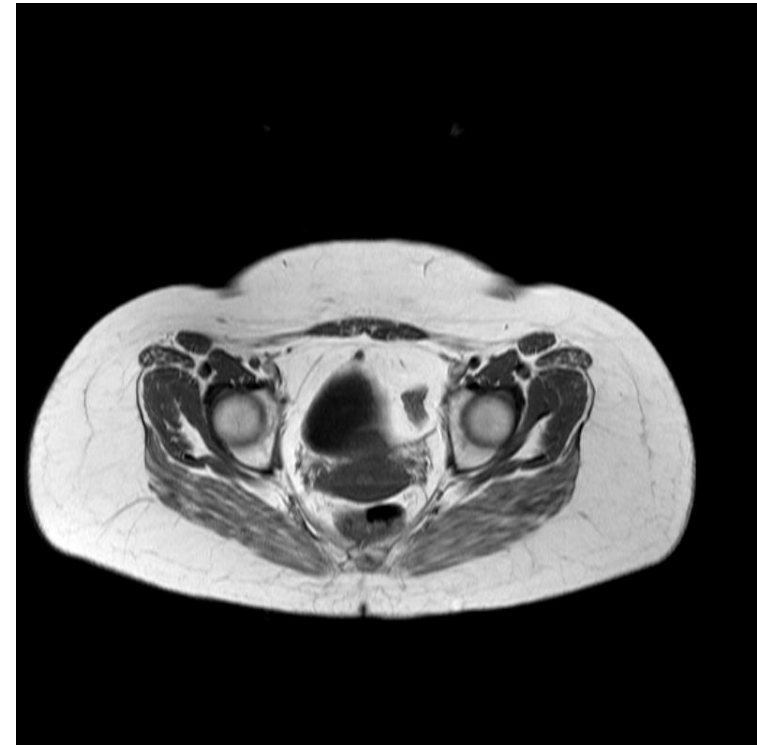

Fig 1. MRI scan of the gluteal muscles showing fatty degeneration and atrophy, T1 sequence.

including p- and c-ANCA, antibodies against the acetylcholine receptor, the MuSK protein and Titinantibodies were unremarkable. We also found regular increase of lactate and ammoniac following physical exercise. Particularly remarkable results were found when testing for the parathyroid function, revealing excessively increased parathyroid hormone (PTH) levels $(959 \mathrm{pg} / \mathrm{ml}$, ref. < 72) along with decreased serum phosphate $(1.8 \mathrm{mg} / \mathrm{dl}$, ref. $2.7-4.5)$ and serum calcium levels $(2.00 \mathrm{mmol} / \mathrm{l}$, ref. 2.08-2.65). Further workup revealed markedly decreased vitamin $\mathrm{D}$ levels $(<4.0$ $\mathrm{ng} / \mathrm{ml}$, ref. 31-100).

Based on these results, we diagnosed secondary hyperparathyroidism with osteomalacia and a neuromuscular disorder as a result of presumably long standing vitamin D deficiency. Further rheumatological workup confirmed the diagnosis of Sjögren's syndrome, but causal relationship with the neuromuscular symptoms was found to be unlikely. Endocrinologists recommended a therapy with high dose vitamin D substitution (single i.m. injection of cholecalciferol 200,000 IE, followed by weekly oral intake of cholecalciferol 20,000 IE) and oral substitution of calcium $2000 \mathrm{mg}$ daily. Three months after the recommended therapy was started, serum calcium levels had normalized and serum PTH levels had decreased to $90 \mathrm{pg} / \mathrm{ml}$. Moreover both neurological symptoms and clinical appearance markedly improved: the patient did not suffer from myalgia anymore and was able to walk without aid again.

\section{Discussion}

We describe a young female patient with slowly progressive weakness and dystrophic changes of the proximal muscles reflecting a structural muscular manifestation of secondary hyperparathyroidism.

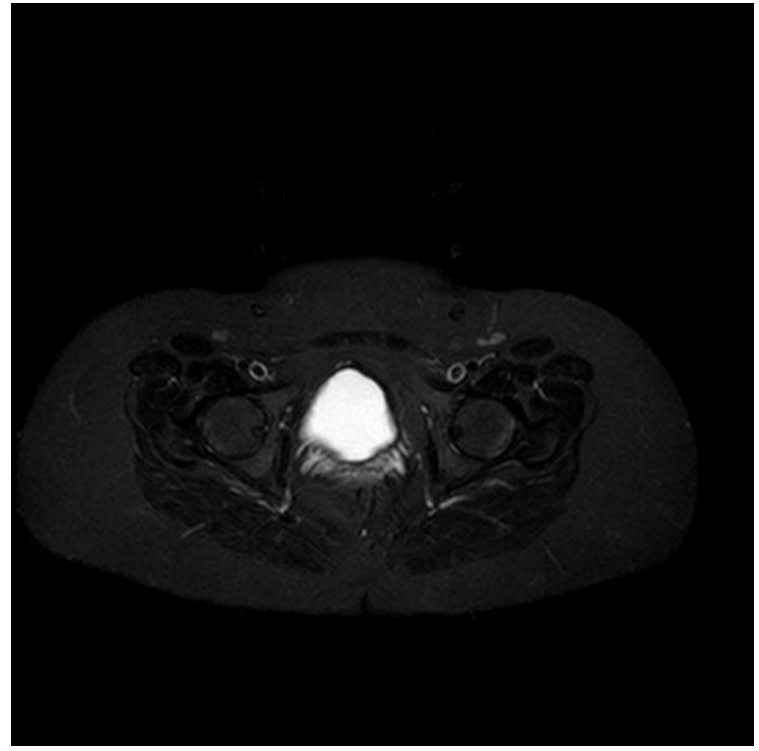

Fig 2. MRI scan of the gluteal muscles, showing fatty degeneration and atrophy, STIR sequence..

Clinical features were mainly consistent with those of a neuromuscular disorder, and the rapid response to vitamin D substitution confirmed the causal relationship to SHPT.

During the past decades only few cases were reported of neuromuscular disorders resulting from hyperparathyroidism. In the 1970ies, Engel and colleagues described several patients with primary or secondary hyperparathyroidism presenting with symptoms of neuromuscular disorders and both EMG and MRI findings consistent with myopathy but absence of myopathic features on muscle biopsy. ${ }^{1,2}$ Both groups (PHPT and SHPT) showed significant improvement of neuromuscular symptoms following treatment of the underlying endocrinopathy. A similar case was reported by Rollinson and Gilligan in the late 1970ies who diagnosed PHPT in a 46-year old woman with a three year history of proximal muscle weakness. ${ }^{3}$ Schneider et al. described a 60 year old woman with a history of PHPT, presenting with typical features of proximal myotonic myopathy (PROMM) and dystrophic changes of the tigh muscles on MRI but no typical myopathic features on muscle biopsy. ${ }^{4}$ Delbridge and colleagues who examined 42 elderly patients undergoing parathyroid surgery found that patients presenting with preoperative neuromuscular symptoms had significantly higher preoperative serum calcium and parathyroid hormone levels compared to those without neuromuscular symptoms, and most of the neuromuscular symptoms improved in the postoperative period. $^{5}$ On the contrary, Chou and colleagues who examined nine patients with PTH and muscular weakness found no correlation of postoperative muscle recovery with preoperative serum calcium, phosphate, alkaline phosphatase and parathyroid hormone levels. ${ }^{6}$ 
While neuromuscular symptoms are common in hypoparathyroidism, neuromuscular presentation in hyperparathyroidism is unusual and has yet been described in few case reports only. The presence of myotonic features which has been described in several reports including the case presented indicates a possible effect of hypocalcemia due to the decreased inhibition of depolarization of nerve and muscle fibers. ${ }^{7}$ Verges and colleagues have discussed a direct action of PTH as the cause of neuromuscular weakness, ${ }^{8}$ however this hasn't been proven so far. Our MRI findings of the proximal muscles were consistent with those reported from similar cases, indicating structural muscular damage as a result of untreated hyperparathyroidsm.

In conclusion, neuromuscular manifestation of HPT should be considered in all cases of unclear progressive muscular weakness, especially as symptoms are likely to improve following treatment of the underlying endocrinopathy.

\section{Author's contributions}

Authors have contributed equally to the manuscript.

\section{Acknowledgments}

The authors report no acknowledgments.

\section{Conflict of Interest}

The authors submit no conflict of interest regarding the publication of this article.

\section{Corresponding Author}

Dr Karim Hajjar, MD

University Hospital Essen, Department of Neurology

Hufelandstrasse 55 - 45147 Essen - Germany

Tel: +49 201723 84495; Fax: +49 2017236981

Email: karim.hajjar@uk-essen.de

\section{E-mail of coAuthor}

Tim Hagenaker: tim.hagenacker@uk-essen.de

\section{References}

1. Patten BM, Bilezikian JP, Mallette LE, et al. Neuromuscular disease in primary hyperparathyroidism. Ann Intern Med 1974;80(2):182-93.

2. Mallette LE, Patten BM, Engel WK. Neuromuscular disease in secondary hyperparathyroidism. Ann Intern Med 1975;82(4):474-83.

3. Rollinson RD, Gilligan BS. Primary hyperparathyroidism presenting as a proximal myopathy. Aust N Z J Med 1977;7(4):420-1.

4. Schneider C, Grimm T, Kress W, et al. Hyperparathyroidism in a patient with proximal myotonic myopathy (PROMM). Neuromuscul Disord 2000;10(7):481-3.

5. Delbridge LW, Marshman D, Reeve TS, et al. Neuromuscular symptoms in elderly patients with hyperparathyroidism: improvement with parathyroid surgery. Med J Aust 1988;149(2):74-6.

6. Chou FF, Sheen-Chen SM, Leong CP. Neuromuscular recovery after parathyroidectomy in primary hyperparathyroidism. Surgery 1995;117(1):18-25.

7. Armstrong CM, Cota G. Calcium block of Na+ channels and its effect on closing rate. Proc Natl Acad Sci U S A 1999;96(7):4154-7.

8. Verges B, Wechsler B, Brunet P, Robin PM, et al. Neuromuscular forms of hyperparathyroidism. Apropos of 2 cases. Ann Med Interne (Paris) 1988;139(4):254-7. [Article in French]. 